Chapter 1

\title{
Surface effects on elastic structures
}

\author{
Hadrien BENSE \\ AMOLF, Science Park 104, 1098 XG Amsterdam, the Netherlands \\ Benoit ROMAN \\ PMMH (CNRS-ESPCI-Sorbonne Université- Université Paris) \\ José BICO \\ PMMH (CNRS-ESPCI-Sorbonne Université- Université Paris)
}

\subsection{Introduction}

Classical continuum elasticity is scale invariant: as long as proportions are kept constant, the size of an object does not matter in regard to elastic deformations. For example, buckling of a structure of a given shape would occur for the same imposed strains, independently of its size. We would therefore expect the same phenomena observable at macroscopic scale to occur at microscale (and maybe even at nano-scale as long as continuum approximation holds).

But the presence of a surface energy $\gamma$ modifies this picture by introducing a lengthscale in the problem. In particular, the coupling between surface interactions and elasticity is characterized by a length scale that compares the force per unit length exerted by the surface effect to the rigidity of the solid: $\ell_{e c}=\gamma / E$, where $E$ is the Young's modulus of the material. In this situation, the size $L$ of the system therefore matters, and a structure bigger than $\ell_{e c}$ is insensitive to surface effect, while a smaller structure might be strongly deformed. In fact we will see that for slender structures, even when $L \gg \ell_{e c}$, large deflections may still occur, because slenderness implies a weak stiffness in bending. 
In this chapter we are interested in how two different surface effects can deform elastic structures. We start with the interaction between capillary surface tension and slender mechanics, and how a droplet can deform a thin sheet. We then focus on electrostatic surface effects, and their use to actuate electroactive polymers membranes.

\subsection{Liquid surface energy}

In this first part we show how the surface tension of a liquid interface in contact with an elastic solid may deform it. Recent years have seen a large body of research effort in this field. Here we will only give scaling arguments and present the consequences of a surface tension forces at micro-scale; thorough reviews are available (see for example ??)

\subsubsection{Can a liquid deform a solid?}

Surface tension is the surface energy cost, $\gamma$, associated to the creation of an interface between two materials (be they liquid or solid). $\gamma$ is positive: surface tension tends to minimise the area of an interface. These capillary interactions are responsible for a large number of phenomena in liquids, such as imbibition, the motion of insects at the surface of water, the spherical shape of small drops and bubbles (?).

In this section, we are interested in how surface tension can deform a solid which is not slender. Consider for example the effect of a droplet deposited on a solid: it exerts

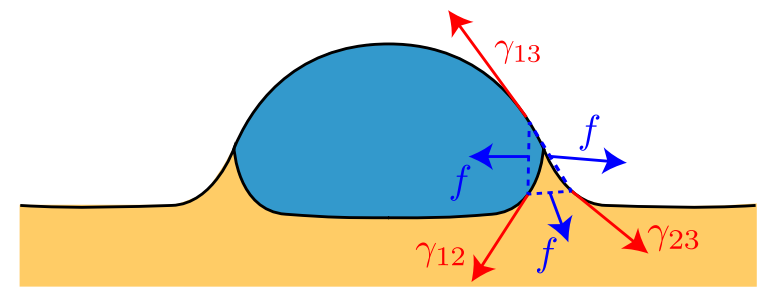

Figure 1.1. Deformation of a solid by a liquid.

a torque on the latter. Indeed the air/liquid surface tension pulls up the solid $\left(\gamma_{13}\right.$ in fig 1.1), while the Laplace pressure pushes down (note that Laplace pressure ensures the vertical equilibrium of forces in the drop, and the horizontal projection leads to the classical Young-Dupré's relation), so that the solid may deform (?). However, taking a standard value of $\gamma \approx 70^{-1} \mathrm{~N} / \mathrm{m}$ for liquid water, and a Young's modulus on the 
order of $70 \mathrm{GPa}$ for the solid (typically glass), one finds that the fluid can deform the solid on a length scale on the order of $\gamma / E \approx 1 \mathrm{pm}$. At such a small scale (smaller than intermolecular distances), the use of continuum mechanics is dubious, and such elasto-capillary effects are irrelevant. But the recent development of microfabrication methods, ultra soft gels and observation techniques have allowed the study of the coupling between capillary forces and elasticity. For example, the deformation near the contact line of a droplet lying on a very deformable substrate $(E \approx 3 \mathrm{kPa})$ has been observed with confocal microscopy. It has been shown that the substrate adopts a shape that does not depend on its thickness, nor on the droplet size, but only on the liquid composition (?. Another example is the rounding off of extremely soft gels (shear modulus between 35 and $350 \mathrm{~Pa}$ ). These gels are casted with sharp angles, but the surface tension of the solid softens the corners so that they exhibit a curvature on the order or $E / \gamma$ (see ?). This experimental observation of the action of a solid's surface tension has lead to many numerical and theoretical developments.

In conclusion, we have seen that a liquid can deform a solid in its bulk, but only at a very small scale, and for very soft solids. However we will see now that on slender structures, surface or capillary effects may produce macroscopic deformations even in materials with a high Young's modulus.

\subsubsection{Slender structures}
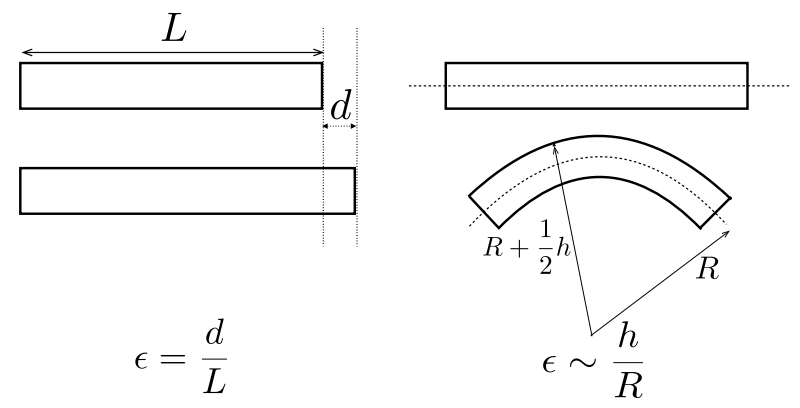

Figure 1.2. The two modes of deformation of a slender structure: stretching (left) and bending (right).

A slender structure is a structure with at least one dimension small when compared to the others. A plate, for example, has a very small thickness $h$ compared to its length $L$ and width $w$. Due to their slenderness, these structures can be thought as having two modes of deformation. On the one hand, they can undergo stretching (or equivalently compression), with an elastic energy

$$
\mathcal{E}_{\text {stretch }} \simeq E h L w \epsilon^{2}
$$


for an in-plane strain $\epsilon$, where $E$ is Young modulus of the plate (Left scheme of figure 1.2).

On the other hand, the plate can be given a radius of curvature $R$. The bending energy associated to this deformation is

$$
\mathcal{E}_{\text {bend }} \simeq \frac{B}{R^{2}} L W,
$$

where $B \simeq E h^{3}$ is the bending stiffness of the plate (right scheme of figure 1.2). Stretching energy is therefore proportional to the thickness $h$, whereas bending energy is proportional to $h^{3}$. As a consequence, the bending energy of a thin structure (small thickness $h$ ) vanishes very quickly when the thickness vanishes. What are the practical consequence of this very compliant mode of deformation?

\subsection{3. wrapping a cylinder}

A simple way to understand how surface forces may bend a slender structure is to consider a solid cylinder of radius $R$, covered with a liquid of surface tension $\gamma$, and a sheet of thickness $h$. We assume the liquid to perfectly wet both solids. What are

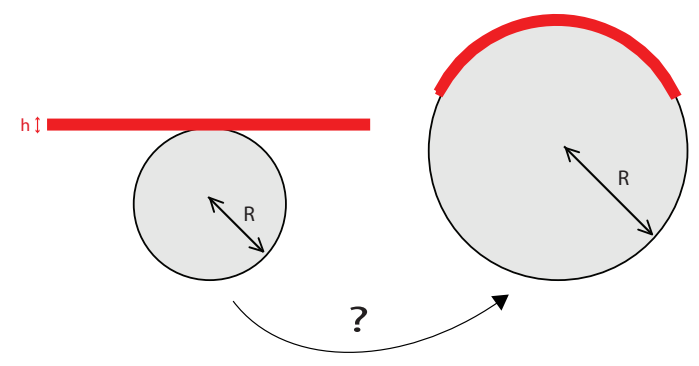

Figure 1.3. Can a thin sheet wrap around a wet cylinder?

the conditions for the sheet to spontaneously wrap the cylinder (fig 1.3)? Wrapping occurs if surface tension is strong enough to bend the sheet with a radius of curvature $R$. This is a pure bending problem, and the energetic cost associated with this deformation is proportional to $E h^{3} \frac{L w}{R^{2}}$. However, covering the cylinder with the sheet reduces the interface between the liquid and the air, and thus is energetically favorable. The associated energetic gain is $2 \gamma L w$. These two energies are in competition, and wrapping will be possible when the energetic gain due the reduction of the air/liquid interface is higher than the energetic cost of the elastic deformation 
of the sheet, i.e when

$$
E \frac{h^{3} L w}{R^{2}}<2 \gamma L w .
$$

This condition can be expressed in terms of a cylinder critical size:

$$
R>\ell_{B}=\sqrt{\frac{E h^{3}}{\gamma}},
$$

where $\ell_{B}$ is the capillary bending length, the relevant length scale when dealing with bending deformations of slender structures by capillary effects. It compares the bending stiffness of the elastic structure to the surface tension of the liquid. If a cylinder has a radius smaller than this length, surface tension will not be able to sufficiently deform the sheet for wrapping to occur.

Elastocapillary deformation is not simply an interesting academic problem, it also has practical consequences in the field of microfabrication. Indeed, the main technique used to manufacture Micro Electro Mechanical Systems (MEMS), or micro-electronic elements is photolithography. After an insulation step, a photo-sensitive resin is put into a solvent solution. During the drying process, capillary bridges may form inside these objects, that can cause deformations, stictions or even fractures. These irreversible events are strong limiting factors in the elaboration of slender micro structures, such as nanolines, micro cantilevers or micro stamps (???).

\subsection{4. capillary origamis}

Manufacturing 3D structures at a microscale with classical microfabrication techniques is a very complicated task, partly because of the limiting role played by surface tension. This force can also be harnessed. The top row of figure 1.4.a presents examples of micrometer cubes obtained thanks to capillary interactions. A liquid metal droplet is deposited at the hinge of an initially flat metallic sheet, as it tends to minimise its interface with the air, it folds the cube (see scheme 1.4.a and ?). We will focus on the technologically simpler case where a macroscopic sheet is deformed by a droplet (figure 1.4.b). What happens when a water droplet is deposited on a thin polymer sheet? Does the drop spread, or does the sheet wrap the droplet ? If, for example the droplet is deposited on a $50 \mu \mathrm{m}$ thin silicone elastomer square sheet we first observe that the corners of the sheet wrap the droplet. As the liquid evaporates the sheet bends more and more, until its complete closing (see pictures in fig. 1.4). Once the droplet is completely evaporated, the sheet may return to its flat state, or remain curved, depending on the intensity of Van der Waals interactions. How does the capillary forces act on the sheet? On the one hand, liquid/air surface tension exerts a traction that pulls the sheet up, while on the other hand, Laplace pressure exerts a pressure that pushes the sheet down (the drop being curved, the pressure 


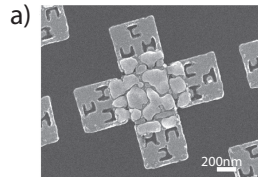

b)

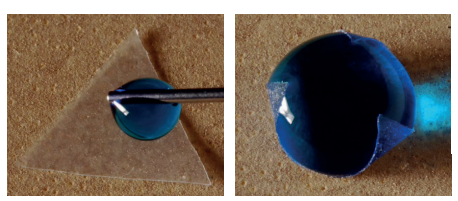

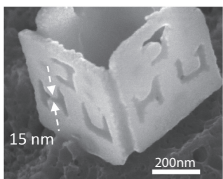

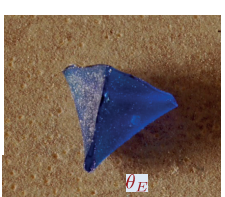

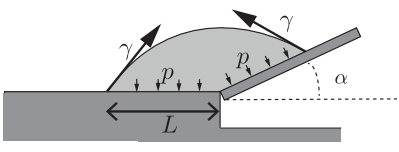

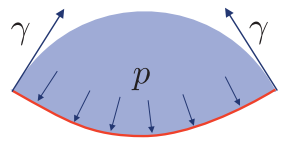

Figure 1.4. a.) Cubic boxes obtained after the fusion of the welding deposited on the hinge of the initial pattern (taken from ?). b.) capillary origami ?

inside the liquid is higher than outside). The drop is therefore exerting a moment on the sheet (scheme fig.1.4.b)). The capillary torque scales as $\gamma L^{2}$, while the typical torque to bend the sheet on it own size scales as $B \simeq E h^{3}$. Therefore, we expect wrapping to happen if the sheet has a size exceeding

$$
L_{c r i t} \simeq \sqrt{\frac{B}{\gamma}} \simeq \ell_{B} .
$$

Experiments have been carried out with sheets of different thicknesses and different shapes (triangles or squares). In each case the critical length has been determined. $L_{c r i t}$ is indeed found to be proportionnal to $\ell_{B}$, with a prefactor that depends on the geometry of the sheet: $L_{\text {crit }} \approx 12 \ell_{B}$ for triangles, $L_{\text {crit }} \approx 7 \ell_{B}$ for squares (see ?).

Interestingly, this law holds up to nanometric scales as shown with simulations on graphene sheets (see ?). At higher scale, gravity starts to play a role, and the maximal size is fixed by the capillary length. Finally, the initial shape of the sheet can be tuned to obtain different 3D shapes, as illustrated in fig 1.5.

In this first part, we studied how a liquid can deform a solid through surface tension interactions. We focused on the interplay between the peculiar mechanics of slender structure and surface tension. The slenderness of the structure is what allows the surface tension to produce remarkable deformation, even though $\gamma / E$ remains very small. As we explained, the addition of surface effects brings new length scales to problems that would otherwise be scale invariant. More precisely, we saw that the relevant length scale when dealing with slender structure interacting with capillary forces is the capillary bending length $\ell_{B}$. This length captures the slender elasticity 

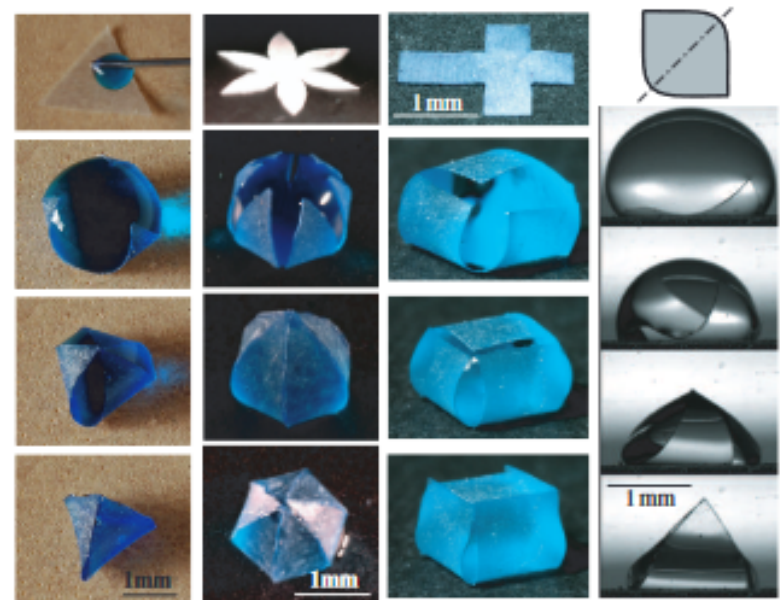

Figure 1.5. Different patterns lead to different $3 D$ shapes

by comparing the bending rigidity to the surface tension. In the following we examine deformation of thin plates, mediated via an electrostatic surface energy.

\subsection{Dielectric Elastomers : a surface effect?}

We now turn to the description of dielectric elastomers, with an emphasis on electrostatic interactions seen as a surface effect, that, as we will show, play an important role.

\subsubsection{Introduction: Electrostatic energy of a capacitor as a surface energy}

These last years have seen the rapid development of a novel class of robotics made of compliant material called "soft robotics". Electro-active polymers are, among the possible technologies to build such robots, a particularly cost effective and easy to manufacture technology. The large deformation electroactive materials can achieve (up to $500 \%$ in area strain (?), and their harmless contact make them suitable for a wide range of potential applications: from bioinspired actuators (??) to soft grippers (??), or tunable lenses (???), and even energy harvesting systems (???). Though their rediscovery is certainly recent, the principle of dielectric actuation can be traced back to Röntgen (?) at the end of the $19^{\text {th }}$ century. The basic idea is indeed fairly simple: a dielectric elastomer is a soft capacitor, in which both electrodes and 
a.
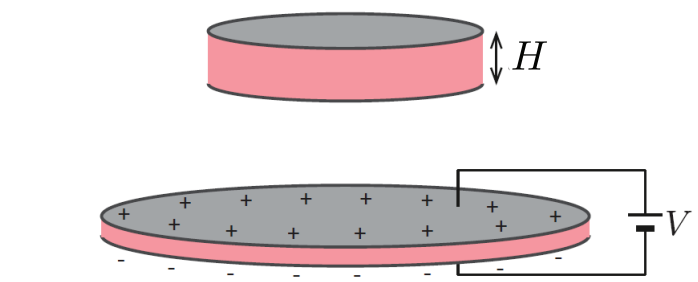

b.
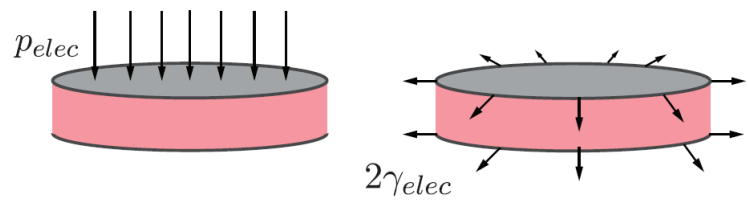

Figure 1.6. a.) An electro-active polymer set-up is made of two compliant electrodes separated by a dielectric polymer. When a voltage is applied, the area of the electrodes increases. b.) The electrostatics interactions can be decomposed in two effects: an electrostatic pressure, acting through the thickness of the membrane, and a negative surface tension, acting along the membrane.

the insulating material can deform under the action of Coulombian interactions (see fig1.6.a). Charges from the opposite faces of the membrane attract each-other, leading to a reduction of its thickness, and therefore to in-plane extension. However a surface effect is also at play in this system. Indeed, the electrostatic energy $\mathcal{E}_{\text {els }}$ of a planar capacitor whose electrodes are separated by $H$ and have a surface $S$ reads:

$$
\mathcal{E}_{\text {els }}=-\frac{1}{2} \varepsilon \frac{V^{2}}{H} S .
$$

where $\varepsilon$ is the permittivity and $V$ the potential difference. For a fixed gap $H$, this energy is proportional to the area of the electrode, and can therefore be seen as a (negative) surface energy, which therefore would tend to increase the surface $S$.

To gain intuition on this surface term, we can evoke electrowetting. Lipmann, during his $\mathrm{PhD}(?)$, imposed a potential difference $V$ between a conductive drop and a metallic surface separated by a dielectric layer (see figure 1.7) with thickness $H$. The drop is observed to spread, and we can interpret this as a result of the repulsion of charges with the same sign at its surface. It is as if the electric field was modifying the drop surface tension $\gamma$ into: $\gamma^{\prime}=\gamma-\frac{1}{2} \varepsilon \frac{V^{2}}{H}$. More precisely the electric field reduces 


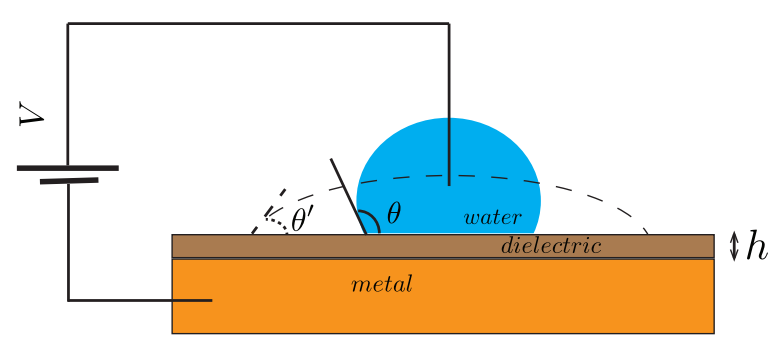

Figure 1.7. Electrowetting: under the application of a voltage, the drop spreads.

the apparent surface tension compared with the initial one, and it therefore seems that its effect can be represented as forces localized at the liquid interface.

In the case of an electro-active polymer (figure 1.6), the material expanding is a solid unlike in the electrowetting experiment, but similar line forces are expected to take place at the boundary of the electrode. We wish to clarify the consequences of such a negative surface tension (with an electrostatic origin) in this elastic solid.

In this chapter, we intend to sketch the formal derivation of the equilibrium equations of a electro-active polymer, with an emphasis on the case where the electrodes are not entirely covering the membrane. In doing so, we will shed light on the practical consequences of the tensile stress existing in the system, and its physical origin, by drawing analogies with capillary surface tension. The rationale developed in this first part will then be applied to an experimental study of a buckling instability triggered by inhomogeneous actuation of the system.

\subsubsection{Mechanics of Dielectric Elastomers}

Our approach will be to derive the equations for the deformation of the polymer under an electric field through a variational approach. We compute both the elastic and the electrostatic energies and minimise the total energy to obtain the equations coupling electrostatics and elasticity. We will then give several interpretations of these equations.

For the sake of simplicity we will consider the planar case of a dielectric strip of thickness $H$, width $W$ and length $L$ at rest. In this reference state, the system is parametrised with the curvilinear abscissa $S$ and the coordinates $X, Y$ and $Z$ running, respectively, along the length, width and thickness of the strip. Upper case letters will refer to the reference, undeformed state, lower case letters to the deformed state. We will assume small strains $e \ll 1$, and a plane strain configuration: $e_{y}=0$ (a plane stress configuration with $\sigma_{y y}=0$ would not change qualitatively the results). We 
summarize here the more detailed derivation reported in ?.

In the deformed state, the curvilinear coordinate is denoted $s$ and $\theta$ is the local slope

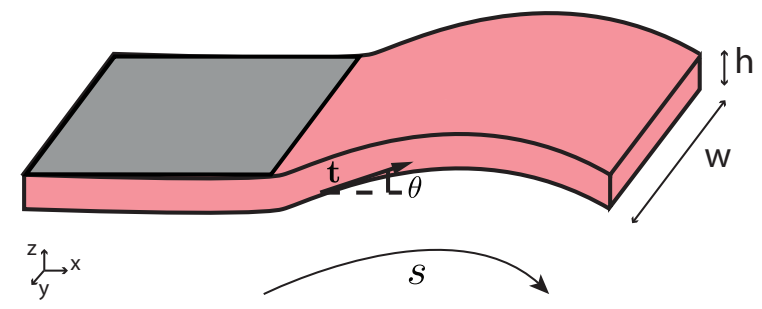

Figure 1.8. Scheme of a partially activated dielectric elastomer. The grey part represents the electrode

(see fig.1.8). We introduce the tangential strain averaged over the thickness of the membrane $e_{t}$, which determines the stretching:

$$
d s=\left(1+e_{t}\right) d S
$$

Similarly, we define the average normal strain $e_{n}$ as:

$$
h=\left(1+e_{n}\right) H
$$

And finally the curvature:

$$
\kappa=\frac{d \theta}{d s}=\frac{1}{1+e_{t}} \frac{d \theta}{d S} \approx \frac{d \theta}{d S}
$$

With the small strains and slender body approximations, the latter indeed means that the curvature is small: $(H \kappa)^{2} \ll 1$. We can therefore neglect any non-linear term involving products of both $\kappa$ and $e_{t}$ or $e_{n}$.

\section{Elastic energy}

In the case of plane strain assumed here (where there is no strain along direction $Y)$, Hooke's law leads to the in-plane stretching energy per unit surface of the strip depends on the tangential and normal strains $\left(e_{t}, e_{n}\right)$, and reads

$$
\mathcal{E}_{\text {stretch }}=\frac{1}{2} \mathcal{Y}\left(e_{t}^{2}+\frac{2 \nu}{1-\nu} e_{n} e_{t}+e_{n}^{2}\right),
$$

where we have introduced the effective stretching modulus

$$
\mathcal{Y}=\frac{(1-\nu) E H}{(1-2 \nu)(1+\nu)}
$$


This expression takes an unusual form, because the variation of thickness $e_{n}$ appears here as an unknown. It is indeed customary in thin plate mechanics to assume that stresses on the faces of the plate vanish, thus setting the value of $e_{n}=-\nu /(1-\nu) e_{t}$ (for this case of plane strain) and $\mathcal{E}_{\text {stretch }}=\frac{1}{2} \frac{E H}{1-\nu^{2}}\left(e_{n}\right)^{2}$. Following this, the in-plane force per unit distance (or tension) $T=\frac{E H}{1-\nu^{2}} e_{t}$ would be proportional to stretching strain, as expected. We will however see that here, electrostatic pressure on the faces is not negligible, and they will play an important role, modifying the relation between tension $T$ and deformation $e_{t}$.

The bending energy per unit surface takes however the usual form

$$
\mathcal{E}_{\text {bend }}=\frac{1}{2} B \theta^{\prime 2}, \text { where the bending stiffness is } B=\frac{E H^{3}}{12\left(1-\nu^{2}\right)} .
$$

\section{Electrostatic energy}

The electrostatic energy of the system comprising the electroactive polymer and the generator imposing a fixed voltage $V$ writes $\mathcal{E}_{\text {els }}=-\frac{1}{2} C V^{2}$. In order to take into account the curvature of the capacitor we consider the capacity of a capacitor formed by two coaxial cylinders:

$$
C=\frac{2 \pi \varepsilon w}{\ln \left(\frac{1+\kappa h / 2}{1-\kappa h / 2}\right)},
$$

with $\varepsilon$ the dielectric constant of the polymer. We expand [1.4] for small curvatures $\kappa h \ll 1$ to find the expression for the electrostatic energy (per unit width) in the slender body approximation:

$$
\mathcal{E}_{\text {els }}=-\int d s \frac{\varepsilon}{2} \frac{V^{2}}{h}\left[1-\frac{1}{12}(h \kappa)^{2}\right] .
$$

This energy is a generalization of equation [1.1] and is still proportional to the surface (remember $\mathcal{E}_{\text {els }}$ above is written per unit width), making it analogous to a surface tension with a negative sign. Moreover, we notice a term proportional to the curvature $\kappa^{2}$, analogous to a elastic bending rigidity in equation [1.3]. The associated electrostatic bending modulus is:

$$
B_{V}=\frac{\varepsilon V^{2} h}{12} \simeq \frac{\varepsilon V^{2} H}{12}
$$

at leading order in strain. Finally, to simplify the following calculations, we express the electrostatic energy in the reference coordinate and as a function of $e_{n}(S), e_{t}(S)$ and $\theta(S)$ :

$$
\mathcal{E}_{\text {els }}=-\frac{\varepsilon}{2} \frac{V^{2}}{H} \int d S \frac{1+e_{t}}{1+e_{n}}+\int d S \frac{1}{2} B_{V} \theta^{\prime 2} .
$$




\section{Variations}

We will assume here, for the sake of simplicity that both end positions of the strip are fixed. The detail of the boundary conditions will not affect the main results of our discussion. The end point position measured from the origin therefore reads:

$$
\mathbf{r}_{\mathrm{end}}=\int d S\left(1+e_{t}\right) \mathbf{t}
$$

where $\mathbf{t}$ is a unit vector tangent to the sheet (having therefore an angle $\theta$ with the $x$ axis). This constraint will be imposed with a Lagrange multiplier $\mathbf{f}$, which will correspond to an externally applied force. The total energy to be minimised is finally

$$
F=\int d S \mathcal{F}\left(e_{t}, e_{n}, \theta\right),
$$

with the surface density of energy given by the sum of all contributions

$$
\begin{aligned}
\mathcal{F}\left(e_{t}, e_{n}, \theta\right)= & \frac{1}{2} \mathcal{Y}\left(e_{t}^{2}+\frac{2 \nu}{1-\nu} e_{n} e_{t}+e_{n}^{2}\right)+\frac{1}{2}\left(B+B_{V}\right) \theta^{\prime 2} \\
& -\frac{\varepsilon}{2} \frac{V^{2}}{H} \frac{1+e_{t}}{1+e_{n}}-\left(1+e_{t}\right) \mathbf{f} \cdot \mathbf{t},
\end{aligned}
$$

where this expression must be used with $B_{V}=0$ and $V=0$ in the portion of the strip that is not covered by the electrode. We now minimise this functional with respect to the different parameters to obtain the set of equilibrium equations:

$$
\begin{aligned}
\frac{\delta F}{\delta \theta(S)} & =-\left(B+B_{V}\right) \theta^{\prime \prime}-\left(1+e_{t}\right) \mathbf{f} \cdot \mathbf{n}=0 \\
\frac{\delta F}{\delta e_{n}(S)} & =\mathcal{Y}\left(e_{n}+\frac{\nu}{1-\nu} e_{t}\right)+\frac{\varepsilon}{2} \frac{V^{2}}{H} \frac{1+e_{t}}{\left(1+e_{n}\right)^{2}}=0 \\
\frac{\delta F}{\delta e_{t}(S)} & =\mathcal{Y}\left(e_{t}+\frac{\nu}{1-\nu} e_{n}\right)-\frac{\varepsilon}{2} \frac{V^{2}}{H}-\mathbf{f} \cdot \mathbf{t}=0 .
\end{aligned}
$$

These equations are geometrically non linear and, as such, are valid for large displacements. In the remaining of the discussion we will consider small strains and so replace $1+e_{n} \approx 1$ and $1+e_{t} \approx 1$.

Equations [1.11] and [1.13] can be interpreted as force balance equations projected, respectively, along the normal and tangential directions. Defining $T=\mathbf{f} \cdot \mathbf{t}$ as the membrane tension (which may be compressive if negative) and $\mathcal{P}$ as the electrostatic 
pressure, defined as the usual attractive pressure between two parallel conducting plates.

$$
\mathcal{P}=\frac{\epsilon}{2} \frac{V^{2}}{H^{2}}
$$

Combining Equations [1.11] and [1.13] to eliminate $e_{t}$, we can obtain the equation governing the tangential strain $e_{t}$ :

$$
T=\frac{E H}{1-\nu^{2}} e_{t}-\frac{1}{1-\nu} H \mathcal{P}
$$

The total tension may be decomposed into the usual elastic tension of the membrane (first term, following the usual Hooke's law), and a compressive (negative) tension induced by electrostatics (second term). Similarly, we can compute the normal strain $e_{n}$, which reads:

$$
\frac{E H}{1-\nu^{2}} e_{n}+\frac{1}{1-\nu} H \mathcal{P}=-\frac{\nu}{1-\nu} T .
$$

Note that equations [1.15] and [1.16] are equally valid in the regions without electrode by taking $\mathcal{P}=0$.

These two equations unveil a first effect of the electro-actuation: at the boundary between the covered and non covered membrane as the electrostatic pressure $\mathcal{P}$ drops to zero, the strains $e_{t}$ and $e_{n}$ are discontinuous. This discontinuity is also naturally observed in the stresses, if computed from these strains using Hooke's law which we will discuss below in section 1.3.2.1.

In equation [1.11] we also recognize the standard Elastica equation (?) for a strip submitted to a force $\mathbf{f}$ at its end :

$$
\left(B+B_{V}\right) \theta^{\prime \prime}+\mathbf{f} \cdot \mathbf{n}=0
$$

with a difference in the added electrostatic bending rigidity $B_{V}$ (valid only in the electroactive region).

The energy minimisation that we performed therefore shows us that the peculiar mode of actuation of this plate does not modify the global buckling equation (apart from an added bending rigidity). Nonetheless, it introduces a discontinuity in the stress and strain fields at the boundary between the electrode and the membrane (although the total force transmitted through the membrane $\mathbf{f}$ is continuous). In the following we propose several interpretations of these equations. 


\subsubsection{Electrostatic surface tension and pressure}

Qualitatively, the electrostatic interactions can be thought as giving rise to two effects. On the one hand, the attraction of opposite sign charges between the faces of the dielectric generates an electrostatic pressure that compresses the membrane along its thickness. This effect can be made apparent by manipulating the force balance along the $\mathbf{z}$ axis (eq [1.16]). Indeed, let us assume for example that no external force is applied to the membrane, i.e $T=0$, then, using Hooke's relation, eq [1.16] can be rewritten

$$
\sigma_{z z}=-\mathcal{P}=-\frac{1}{2} \frac{\varepsilon V^{2}}{h^{2}} .
$$

This compressive stress of electrostatic origin is the classical Maxwell pressure that acts on a rigid plate capacitor. Through Poisson effect, this pressure contributes to the extension of the membrane.

On the other hand, repulsion of same sign charges on each electrode creates a tensile stress. Similarly eq [1.15], equilibrium of longitudinal forces, can be rewritten in absence of externally applied membrane tension $T=0$,

$$
\sigma_{x x}=\mathcal{P}=\frac{1}{2} \frac{\varepsilon V^{2}}{h^{2}} .
$$

An electrostatic positive (tensile) stress, of equal magnitude as the electrostatic pressure, acts along the electrode and helps its expansion. In the end, the system undergoes a compressive stress across its thickness, and a tensile stress along its electrode (see fig. 1.6), leading to the strain derived in equations [1.15] and [1.16]. Interestingly, both effects have the same magnitude.

We may now comment the discontinuities in the elastic stresses at the electrode boundary, for a strip submitted to a total membrane force $T$. The discontinuity in $\sigma_{z z}$ simply results from the fact that an electrostatic pressure $\sigma_{z z}=\mathcal{P}$ is applied under the electrode, whereas $\sigma_{z z}=0$ outside, where the electrostatic pressure vanishes. The situation is more subtle for the in-plane stresses $\sigma_{x x}$. Applying [1.15] inside and outside the electrode and using Hooke's law, we can deduce the value of the elastic stresses jump $\Delta \sigma_{x x}=\mathcal{P}=\frac{\epsilon}{2} \frac{V^{2}}{H^{2}}$. This jump results from a localized force at the boundary of the electrode $\frac{\epsilon}{2} \frac{V^{2}}{H}$, consistently with the negative surface tension deduced from [1.1].

We conclude that elastic stress in the material results from (i) an electrostatic pressure on its faces, and (ii) a surface tension forces acting on the boundaries of the electrode. We propose different interpretations of these stress distributions in the two next sections.

\subsubsection{Doubled electrostatic pressure}

A very common approach to describe electroactive polymers was introduced by Ronald Pelrine in his seminal work (?). To understand it, let us consider a strip 
subjected to a simple mechanical pressure $\sigma_{z z}=-\mathcal{P}_{0}$ on one specific region. In that case, Hooke relations lead to:

$$
T=H w\left(\frac{1}{1-\nu^{2}} E e_{t}-\frac{\nu}{1-\nu} \mathcal{P}\right)
$$

If we now compare eq [1.15] (electrostatic actuation) and eq [1.18] (mechanical actuation), we notice that both equations are equivalent (i.e electrostatic and mechanical actuations produce the same strain) if $\mathcal{P}_{0}=\mathcal{P} / \nu$. In the common case where the dielectric polymer is an elastomer, we have $\nu=0.5$. For a electro-active elastomer, the state of strain under actuation can therefore be derived by considering that a doubled electrostatic pressure acts on the system, without any negative surface tension effect. Our variational approach allows to get convinced that this point of view is still valid in the case where the electrodes do not entirely cover the membrane. This approach is simpler when one is interested in the strain field in the membrane. However, the state of stress, and the added electrostatic bending rigidity are missed.

\subsubsection{Equivalent growth}

Equations [1.15] and [1.16] can be rewritten as :

$$
\begin{aligned}
T & =\frac{E H}{1-\nu^{2}}\left(e_{t}-e_{t}^{0}\right) \\
\frac{E H}{1-\nu^{2}}\left(e_{n}-e_{n}^{0}\right) & =-\frac{\nu}{1-\nu} T,
\end{aligned}
$$

if we define

$$
e_{t}^{0}=(1+\nu) \frac{\mathcal{P}}{E} ; \quad e_{n}^{0}=-(1+\nu) \frac{\mathcal{P}}{E} .
$$

Written in this way, these equations are exactly the ones describing an elastic strip whose reference state differs by a strain $\left(e_{t}^{0}, e_{n}^{0}\right)$ from the initial stress-free reference sate. By reference state we mean the state attained when there is no external mechanical loading $T=0$. This rewriting therefore provides another interpretation of the electrostatic loading: applying a voltage is equivalent to defining a new stress-free reference state for the membrane. This situation is similar to cases of inelastic strain, such as those produced by growth or plastic deformation, which effectively redefines the reference state. Hence, the electrostatic loading can be seen as a modification of the rest length of the strip, together with providing a new bending rigidity $B+B_{V}$. This interpretation differs from other common approaches such as described earlier. It presents the advantage of completely capturing the electrostatic effects, and provide a useful framework when dealing with non-homogeneous actuation. 


\subsubsection{Conclusion and order of magnitude}

In this section, we demonstrated how the electrostatic loading can be decomposed in two effects, first a compressive pressure

$$
\mathcal{P}=\frac{1}{2} \frac{\varepsilon V^{2}}{H^{2}}
$$

on the faces of the electrode, and second a (negative) surface tension

$$
\gamma=-\frac{1}{2} \frac{\varepsilon V^{2}}{H} .
$$

on the electrode. Both effects tend to expand the membrane, with the same order of magnitude (in fact for $\nu=1 / 2$, both effect produce the exact same strain).

Coming back to the introduction of the chapter, we may estimate the effect of say the surface tension term by computing the electrostatic equivalent to the bulk elasto-capillary length $\gamma / E$, in which $\gamma$ designates the electrostatic surface tension defined in [1.22]. $\gamma=\frac{\varepsilon}{E} \frac{V^{2}}{H}$. In typical experiments, we find $\gamma / E=H \mathcal{P} / E \approx 10^{-6} \mathrm{~m}$. As for the capillary case, this length scale is too small to play an important role in the problem. In fact $\mathcal{P} / E$ can be interpreted as the typical strains in the material, which remain modest and do not induce large shape change.

However we will now see through the detail study of a specific example that mechanical instabilities can be harnessed in an electro-actuated thin membrane to obtain interesting shape-changes.

\subsubsection{Buckling experiments}

We investigate experimentally how a non-uniform spatial distribution of voltage can trigger out-of-plane buckling patterns in electro-activated polymers. This work is inspired by non-uniform growth of plant leaves, or material swelling that leads to complex 3D shapes (????).

We focus on a model axisymmetric configuration where a circular membrane floats freely on a bath of water. A compliant circular electrode is deposited in its center, surrounded by passive material (see figure 1.9). When submitted to a voltage, the area of this region tends to increase, up to a point where a buckling instability occurs (see bottom picture in fig 1.9). Actuation is here non-homogeneous since one region is subjected to voltage while the rest of the membrane is not. We use linear elasticity and weakly non-linear equations of thin plates within the framework previously developped to investigate the buckled morphologies. 


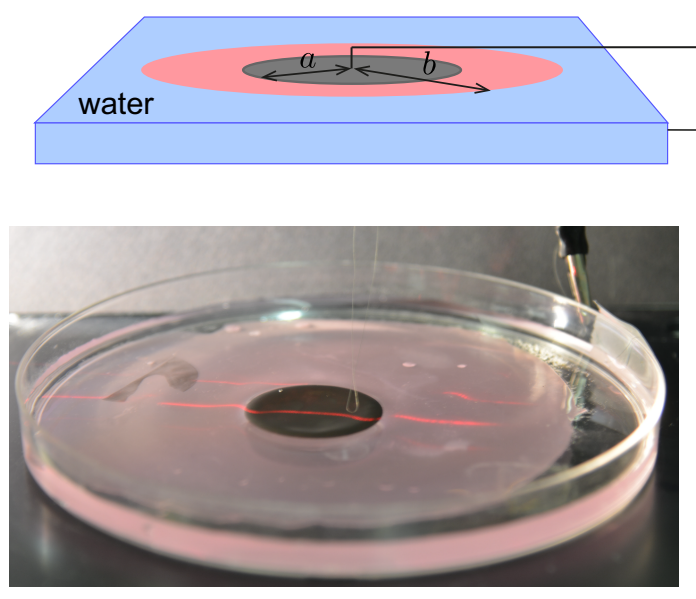

Figure 1.9. Sketch and picture of the set-up. A membrane of PVS floats freely on a bath of soapy water. A circular electrode (black circle) of radius $a$ is connected to a high voltage amplifier, water is connected to the ground. When a threshold voltage has been reached a buckling instability occurs, as can be seen from the deflected laser line on the picture.

\subsubsection{Description of the experiment}

\section{Dielectric membranes}

We use dielectric membranes made of polyvinil siloxane elastomers, of Young modulus $E=250 \pm 15 \mathrm{kPa}$, and dielectric permittivity $\varepsilon_{r}=2.5 \pm 0.6$. The polymer is spin-coated before its reticulation is over to obtain a circular membrane, of radius $b$ and thickness $H$ ranging from 100 to $300 \mu \mathrm{m}$. The compliant electrode consists of carbon black powder manually brushed through a circular stencil of radius $a$ on the surface of the cured polymer. We will refer to the part covered with the electrode as the active part, whereas the uncovered membrane will be designated as the passive part. This powder strongly adheres to the polymer and thus provides an electrical conductivity even when the membrane is strained at $40 \%$ (the strains we achieve experimentally are on the order of $10 \%$.

\section{experimental set-up}

Once prepared, the membrane is gently deposited at the surface of soapy water, where it floats freely. Surfactants allow us to impose a controlled value of the surface tension $\gamma \approx 30 \mathrm{mN} \cdot \mathrm{m}^{-1}$, and enhance the electric conductivity of water. The voltage is imposed through a thin metallic wire (of radius $10 \mu \mathrm{m}$ ) in light contact with the 
circular electrode. The wire is connected to a high voltage amplifier, driven by a signal generator. Water is connected to the ground and plays the role of a second compliant electrode. Voltages applied to the system typically range from $200 \mathrm{~V}$ to $5 \mathrm{kV}$. Out-ofplane deformations are measured with a laser sheet with a grazing incidence projected on the active part of the membrane. The deflection of the laser is recorded using a camera above the set-up, and is directly proportional to the vertical displacement of the membrane. The voltage is increased of $100 \mathrm{~V}$ every $30 \mathrm{~s}$ so that the experiment can be considered as quasistatic, and any viscoelastic effect of the polymer can be neglected.

\subsubsection{Membrane stresses below the buckling threshold}

What is the state of stress in the actuated membrane prior to buckling? We start with accounting for the effect of water surface tension. It induces a tensile strain on the order of $\gamma / E H \approx 10^{-2}$ for a $200 \mu m$ thick membrane with free edges. We showed previously how the electrostatic actuation could be modelled by an equivalent growth. Adapting equation [1.21] to this axisymmetric situation, the electrostatic actuation imposes a new rest length to the electrode, with an extensional strain

$$
e_{0}=\frac{1}{2} \frac{\varepsilon}{E}\left(\frac{V}{H}\right)^{2}
$$

If we now express the strain imposed by the water surface tension, we find an equivalent voltage of $2 \gamma \mathrm{H} / \varepsilon=700 \mathrm{~V}$. This contribution is not negligible. However as we are here interested in the regime below buckling, mechanics remains linear. Surface tension effects and electro-actuation are simply additive and we therefore consider the pre-strained state as the reference state. Water surface tension will be taken into account when we focus on buckling and post-buckling.

The problem is axisymmetric, the mechanical equilibrium reads:

$$
\frac{\partial r \sigma_{r}}{\partial r}-\sigma_{\theta}=0
$$

with $(r, \theta)$ the cylindrical coordinates, with origin the center of the membrane. $\sigma_{r}$ and $\sigma_{\theta}$ are the radial and azimuthal stresses. In our equivalent growth approach, Hooke relations write:

$$
\begin{aligned}
& \sigma_{r}=E^{*}\left(\left(e_{r}-e_{0}\right)+\nu\left(e_{\theta}-e_{0}\right)\right) \\
& \sigma_{\theta}=E^{*}\left(\left(e_{\theta}-e_{0}\right)+\nu\left(e_{r}-e_{0}\right)\right),
\end{aligned}
$$


with $E^{*}=E /\left(1-\nu^{2}\right)$, and $e_{0}=0$ in the passive region. Finally, noticing that radial and azimuthal strains are related to the radial displacement $u(r)$ through $e_{r}=d u / d r$ and $e_{\theta}=r / r$, Equation [1.24] can be rewritten as:

$$
r^{2} u^{\prime \prime}+r u^{\prime}-u=0
$$

where .' means derivation with respect to $r$. The symmetry of the problem imposes $u(0)=0$ and $\sigma_{r}(b)=0$, while the equilibrium conditions imply the continuity of $\sigma_{r}$ and $u$ at the interface between the active and passive regions. With these conditions, equations [1.27] can be solved analytically to obtain the following expressions for the stress:

$$
\begin{aligned}
& \sigma_{r}^{A}=\sigma_{\theta}^{A}=\frac{-\mathcal{P}}{2}\left(1-\frac{a^{2}}{b^{2}}\right)<0 \\
& \sigma_{\theta}^{P}=\frac{\mathcal{P}}{2} \frac{a^{2}}{b^{2}}\left(1+\frac{b^{2}}{r^{2}}\right)>0
\end{aligned}
$$

With $\mathcal{P}=\frac{1}{2} \varepsilon\left(\frac{V}{H}\right)^{2}$. In the active region, both radial and azimuthal stresses are compressive, whereas in the passive region only radial stresses are compressive and the azimuthal stresses are tensile. Both stresses decaying away from the active region (notice also the strong discontinuity in orthoradial stress at $r=a$ ).

\subsubsection{Out of plane buckling}

As voltage is increased, the active region expands, in conflict with the passive region, and we showed that radial compressive stresses build up along the whole membrane. When this stress reaches a high enough value, the membrane undergoes axisymmetric buckling. In contrast with Euler buckling, the out-of-plane deformation is strongly localised in the active region. We also notice that the global mode of the instability depends on the size of the active zone (see figure 1.10a,b). This mode, however, does not depend on the magnitude of the actuation. The superposition of the deflected laser line at different voltages in figure 1.10 clearly shows that increasing the voltage beyond the buckling threshold only increases the amplitude of the deflection.

The water foundation underneath the membrane introduces a new lengthscale that comes from the competition between gravity, and bending stiffness of the plate. Balancing both effects leads to an elastrogravity lengthscale (?, ?): $\ell_{e g}=2 \pi\left(\frac{E H^{3}}{12\left(1-\nu^{2}\right) \rho g}\right)^{1 / 4}$, with $\rho$ the volumetric mass of the water, and $g$ the acceleration of gravity. For a typical membrane of $H=200 \mu \mathrm{m}$, we obtain $\ell_{e g} \approx 1.4 \mathrm{~cm}$. In figure 1.10.a we use $\ell_{e g}$ as a scale bar. when $a \gg \ell_{e g}$ we observe 


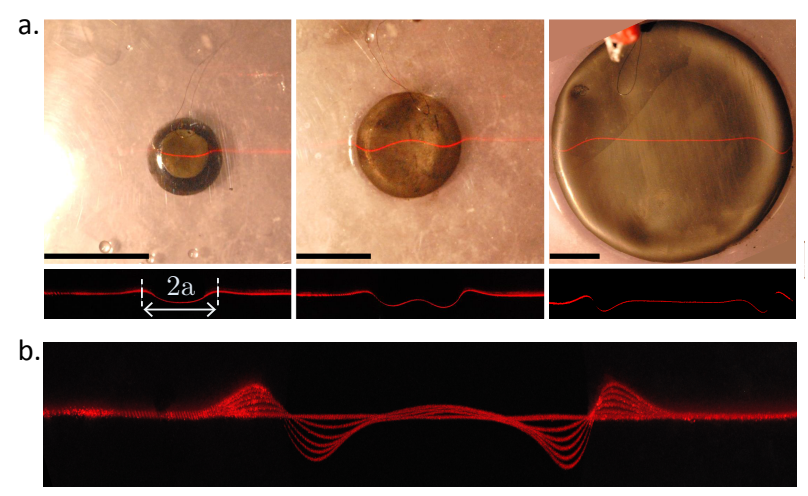

Figure 1.10. a.) Different buckling modes at $V=5 \mathrm{kV}$ and $H=210 \mu \mathrm{m}$ for different radii of the active zone (from left to right: $a=0.5 \mathrm{~cm}$, $a=1 \mathrm{~cm}, a=3 \mathrm{~cm}$. The upper row is a picture of the membrane taken from above, the lower one highlights the profile of the membrane. The scale bar is the elastogravity lengthscale $\left(\ell_{e g} \approx 1.4 \mathrm{~cm}\right) . b$.) Superposition of laser profiles obtained for increasing applied voltage (from 0 to $5 \mathrm{kV}, H=210 \mu \mathrm{m}, a=1.5 \mathrm{~cm}$ ). The buckling mode does not change, only its amplitude increases.

that the buckling is localised near the edges of the electrode, and the width of the corresponding annulus is comparable with this lengthscale (see figure 1.10.b middle and right pictures). Conversely, when $a \leq \ell_{e g}$, the whole active region is deformed ( figure 1.10.a, left picture). We assess the onset of the buckling threshold and its evolution by monitoring the profile as a function of the applied voltage (figure 1.10). We have seen that electrostatic loading produces an actuation strain $e_{0}$ in [1.21], that we compare with the critical compressive strain for the buckling of a 1D strip lying on water $e_{1 D}^{c}=\frac{2 \pi^{2}}{3}\left(h / \ell_{e g}\right)^{2}$. Results are presented in figure 1.11 . We observe a clear increase of the amplitude once a certain threshold voltage has been reached. This evolution is however not as sharp as in the case of a classical pitchfork bifurcation. The smoothness of the experimental transition is probably a consequence of imperfections. Among them we can mention the slight deformation caused by the contact with the wire, and the possible migration of charges outside the active region. To describe the buckling and the post-buckling behaviour, we use axisymmetric weakly non-linear plate equations. We assume that the plate keeps a uniform thickness (strains remain low in our experiments). We use the Föppl-Von Kármán framework. We are therefore left with the resolution of a system of two differential equations: the in-plane equilibrium and the torque equilibrium. We do not take into account the added electrostatic bending rigidity $B_{V}$ that we evidenced previously. Indeed, in this particular case, the total bending rigidity is only modified by $10 \%$ for 
a.

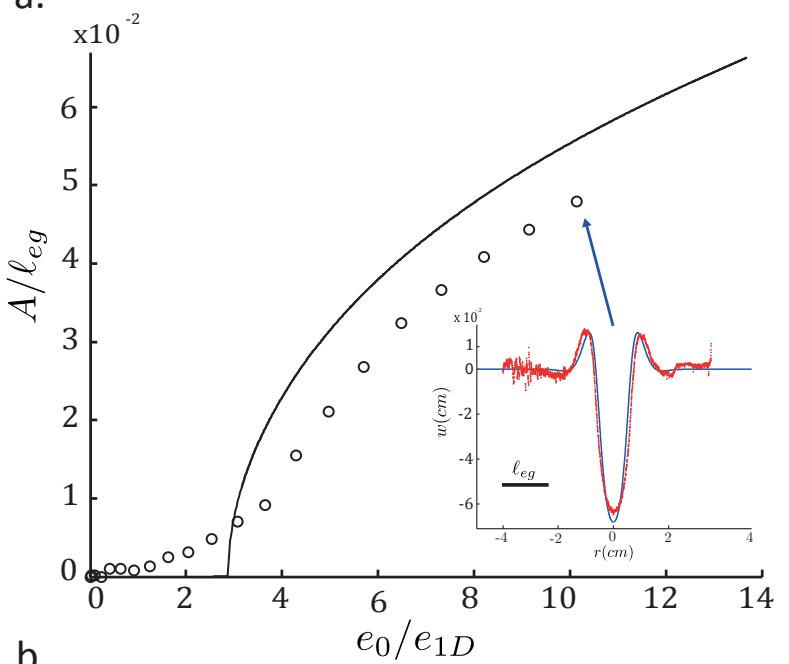

b.

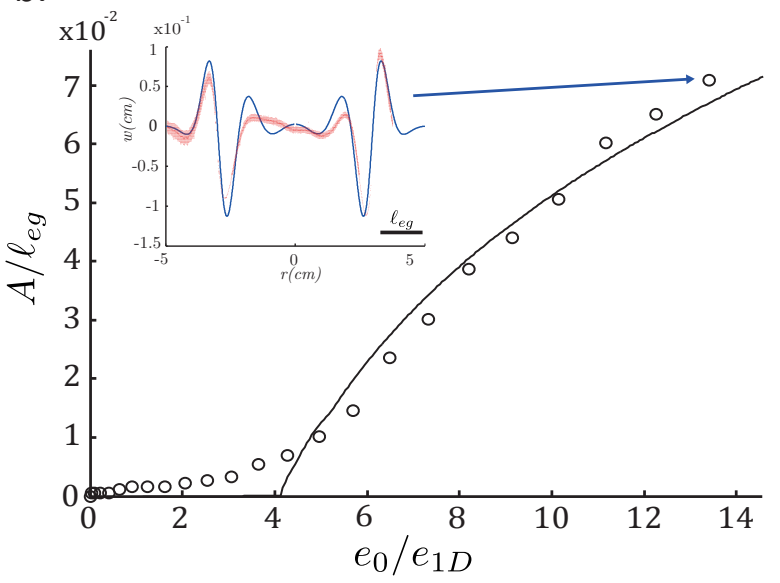

Figure 1.11. Maximal amplitude $A$ of the deflection normalised by $\ell_{e g}$ as a function of $e_{o} / e_{1 D}$, where $e_{o}$ is the expansion strain triggered in the active zone by electrostatic loading, and $e_{1 D}$ is the typical compressive strain leading to buckling of a 1D strip floating on water. Experimental data (circles) are compared to numerical integration of equations [1.30], [1.31] (continuous line). No fitting parameter. Inset: superposition of experimental (red) and numerical (profiles) at the point indicated by the arrow. a.) $a=6 \mathrm{~mm}$. b.) $a=30 \mathrm{~mm}$ 
the highest voltage accessible in our experiments. In the axisymmetric regime, the equations read

$$
\begin{aligned}
& r^{2} u^{\prime \prime}+r u^{\prime}-u+\frac{1-\nu}{2} r w^{2}+r^{2} w^{\prime} w^{\prime \prime}=0 . \\
& B \Delta^{2} w=N_{r} w^{\prime \prime}+N_{\theta} \frac{w^{\prime}}{r}+q
\end{aligned}
$$

With $N_{r}=H \sigma_{r}$ and $N_{\theta}=H \sigma_{\theta}$ and boundary conditions $w^{\prime}(0)=w^{\prime \prime}(0)=0$, $N_{r}(b)=\gamma, w^{\prime \prime}(0)=0$ '. Experiments and numerics are in fairly good agreement, showing the relevance of our modeling to capture this buckling instability.

\subsubsection{Conclusion on dielectric elastomers}

We introduced a framework for dealing with electrostatic surface effect. In particular, we drew an analogy with biological growth. Building on this analogy we showed how a non-uniform voltage distribution can trigger buckling instability in free floating electro-active elastomer sheet. We saw that the buckling mode depends on the size of the electrode, and is set by an interplay between hydrostatics and the bending stiffness of the membrane. We also demonstrated how the framework developed in the first part, coupled with classical weakly non-linear plate equations allows us to capture well the behaviour of the system. This tool can then be used to study the influence of the size of the electrode on the buckling threshold of the system (see ?)

This study represents a simple demonstration of how inhomogeneous growth can trigger 3D shapes in electro-active polymer. Building on this idea, more complicated electrode geometries have been considered, that lead to different buckling modes ??. In parallel, numerical tools are developed to study this type of problems?.

\subsection{Conclusion}

In this chapter we discussed the effects of two different interfacial energies, one of capillary nature, the other of electrostatic nature. On the one hand Capillary surface tension stems from the energetic cost of creating an interface (due to molecular interaction at the interface). On the other hand, an "electrostatic surface tension" can be associated to electrostatic repulsion along the surface : charges on the edge of the electrode are pushed further away by their neighbours. This analogy also helps us understand that the effects of these surface forces are opposite: capillary surface tension tends to limit the size of the interface, while, conversely electrostatic effects favour the expansion of the electrode. 
The existence of a surface energy $\gamma$ brings a new length-scale in elasticity $\ell_{e c}=\gamma / E$. In particular, we introduced the elasto-capillary (with $\gamma$ the liquid-vapour surface energy) and elasto-electro (with $\gamma=\frac{1}{2} \varepsilon V^{2} / H$ ) lengths that both demonstrate how small the deformation produced by these surface effects are in general, even for relatively soft elastomers (a few hundreds of $k P a$. Indeed $\gamma / E$ typically ranges from $10^{-7} \mathrm{~m}$ to $10^{-5} \mathrm{~m}$. It is clear that elastomer structures smaller or comparable to this length are prone to large deformations, either by capillary or electrostatic forces. For structures made of a stiff materials, we do not expect capillary nor electrostatic surface energies to generate large strains, even at micro-scale, because $\gamma / E$ is on the order of a picometer, smaller than the inter-atomic distance.

Nevertheless we have shown that in slender structures the effect of small deformations may be amplified greatly. In such problems, a bending elasto-capillary length $\ell_{B}=\sqrt{B / \gamma}$, where $B$ is the bending stiffness is more relevant. When $\ell_{b}$ becomes on the order of the size of the system, surface forces are expected to generate strong bending: we showed how a droplet may fold a thin plate, and form 3D origamis through capillary forces tending to reduce the droplet surface. In contrast, electrostatic surface tension generates an expansion of the electrode. We presented a case of inhomogeneous actuation that triggers a buckling instability in a macroscopic thin plate. In both the capillary $\left(\ell_{B} \sim h^{3 / 2}\right)$ and the electrostatic case $\left(\ell_{B} \sim h^{2}\right)$, the typical radius of curvature induced by surface forces $l_{B}$ vanishes faster than the thickness $h$ when a structure is scaled down: eventually, this radius of curvature becomes of the order of the structure size, leading to significant bending. We conclude that both types of surface effects become all the more important when micro or nanostructures are considered. Such effects could be used to manipulate or actuate efficiently micro-structures.

\subsection{Bibliography}

A.N. Norris, A. C., Sheng, P. (1985), J. Mech. Phys., 33, 525-543.

Batchelor, G. (1970), title, J. Fluid Mech., 44, 545-570.

Cox, R. (1971), J. Fluid Mech., 45, 625-657.

Dai, S.-C., Bertevas, E., Qi, F., Tanner, R. I. (2013), Viscometric functions for noncolloidal sphere suspensions with Newtonian matrices, Journal of Rheology, 57(2), 493-510.

Doi, M., Edwards, S. (1988), The Theory of Polymer Dynamics, Oxford University Press, Oxford.

Einstein, A. (1956), Investigations on the Theory of the Brownian Movement, Ed. R. Fürth, R., New York.

Ericksen, J. (1960), Kolloid-Z., 173, 117-122.

Folgar, F., Tucker, C. (1984), J. Reinforced Plast. Compos., 3, 98-119. 
Foss, D., Brady, J. (2000), title, J. Fluid Mech., 407, 167.

Gadala-Maria, F., Acrivos, A. (1980), Shear-induced structure in a concentrated suspension of solid spheres, Journal of Rheology, 24(6), 799-814.

Goddard, J., Miller, C. (1967), , 28, 657.

Hand, G. (1961), Arch Ration Mech Anal, 7, 81-86.

Happel, J., Brenner, H. (1973), Low Reynolds Number Hydrodynamics, Noordhoff International Publishing, Leyden.

Hinch, E., Leal, L. (1972), J. Fluid Mech., 52, 683-712.

Jeffery, G. (1922), Proc. Roy. Soc. Lond. A, 102, 161-179.

Kim, S.and Karrila, S. (1991), Microhydrodynamics: Principles and Selected Applications, Butterworth and Heinemann, Boston.

Landau, L., Lifshitz, E. (1959), Fluid Mechanics, Pergamon Press, New York.

Leal, L., Hinch, E. (1973), Rheologica Acta, 12, 127-132.

Lin, Y., Phan-Thien, N., Cheong Khoo, B. (2014), Normal stress differences behavior of polymeric particle suspension in shear flow, Journal of Rheology, 58(1), 223235.

Lyon, M. K., Mead, D. W., Elliott, R. E., Leal, L. G. (2001), Structure formation in moderately concentrated viscoelastic suspensions in simple shear flow, Journal of Rheology, 45(4), 881-890.

Mall-Gleissle, S., Gleissle, W., McKinley, G., Buggisch, H. (2002), The normal stress behaviour of suspensions with viscoelastic matrix fluids, Rheologica Acta, 41(12), 61-76.

Metzner, A. B. (1985), Rheology of suspensions in polymeric liquids, Journal of Rheology, 29(6), 739-775.

Mewis, J., Wagner, N. (2012), Colloidal Suspension Rheology, Cambridge University Press, New York.

Morris, J. (2009), title, Rheologica Acta, 48, 909-923.

Norris, A. (1985), Mech. Mat., 4, 1-16.

Pan, W., Caswell, B., Karniadakis, G. E. (2010), Rheology, Microstructure and Migration in Brownian Colloidal Suspensions, LANGMUIR, 26(1), 133-142.

Phan-Thien, N., Fan, X.-J., Khoo, B. (1999), Rheologica Acta, 38, 297-304.

Phan-Thien, N., Kim, S. (1994), Microstructure in Elastic Media Principles and Computational Methods, Oxford University Press, New York.

Phan-Thien, N., Pham, D. (1997), Differential multiphase models for polydispersed suspensions and particulate solids, Journal of Non-Newtonian Fluid Mechanics, 72(2-3), $305-318$.

Roscoe, R. (1952), British J. Appl. Phys., 3, 267-269. 
Tanner, R. I., Qi, F., Housiadas, K. D. (2010), A differential model for the rheological properties of concentrated suspensions with weakly viscoelastic matrices, Rheologica Acta, 49(2), 169-176.

Zarraga, I. E., Hill, D. A., Leighton, D. T. (2000), The characterization of the total stress of concentrated suspensions of noncolloidal spheres in newtonian fluids, Journal of Rheology, 44(2), 185-220.

Zarraga, I. E., Hill, D. A., Leighton, D. T. (2001), Normal stresses and free surface deformation in concentrated suspensions of noncolloidal spheres in a viscoelastic fluid, Journal of Rheology, 45(5), 1065-1084. 
